\title{
COULD MATERNAL AUTOANTIBODIES PLAY AN ETIOLOGICAL ROLE IN ASD DEVELOPMENT?
}

\author{
Dudova $^{1}$, J. Ziak², M. Balastik², P. Buran², M. Janikova3 , A. Stuchlik ${ }^{3}$, \\ K. Horackova ${ }^{4}$, M. Hrdlicka'1. \\ ${ }^{1}$ Charles University Second Faculty of Medicine and Motol University Hospital, Dpt. of Child Psychiatry, Prague, Czech \\ Republic. ${ }^{2}$ Institute of Physiology of the Czech Academy of Sciences, Dpt. of Molecular Neurobiology, Prague, Czech \\ Republic. Institute of Physiology of the Czech Academy of Sciences, Dpt. of Neurophysiology of the Memory, Prague, \\ Czech Republic. ${ }^{4}$ Charles University First Faculty of Medicine, Dpt. of Psychiatry, Prague, Czech Republic.
}

\section{Objectives:}

Autism spectrum disorders (ASD) are considered to have multiple causes, which are so far largely unknown. Recent data demonstrate that an important role in ASD development is played by immune system and that a significant number of mothers of ASD children carries maternal autoantibodies directed against various fetal brain proteins. The role of the maternal autoantibodies in pathogenesis of ASD has not been clarified yet. Collapsin response mediator proteins (CRMP2 and CRMP1) which integrateing multiple signaling cascades regulating neuron growth and migration are amongst the main epitopes targeted by maternal autoantibodies.

\section{Background:}

Targeting of CRMP2 or CRMP1 by maternal autoantibodies could change the levels or distribution of these proteins in the developing nervous system leading to deficits in axon growth, cortical migration or dendritic projection. Aims of the project are to explore at multiple systemic levels the causative role of CRMP2 signaling and isoform-specific CRMP2 autoantibodies in the pathogenesis of ASD using human samples and novel animal models.

\section{Methods}

To date, sera were collected from mothers of ASD patients $(n=46)$ and age-matched healthy controls $(n=35)$ and presence of isoform specific antibodies against CRMP2 and CRMP1 was analyzed by Western blotting and ELISA assay. Mouse model of CRMP2 deficiency (CRMP2 KO mice) was generated by TALEN mutagenesis. Immunohistological analysis of the developing brain was performed in CRMP2 KO mice together with behavioral analysis in the early postnatal as well as adult stages (e.g. ultrasonic vocalization, cognitive test).

\section{Preliminary results}

We found increased prevalence of CRMP1 and CRMP2 isoform-specific antibodies in maternal sera of ASD patients.

We demonstrate that CRMP2 deficiency in mice leads to multiple histological changes as well as to changes of neuron cytoarchitecture (hippocampal dendritic spine densities) previously associated with ASD pathogenesis. Moreover CRMP2 KO mice show early postnatal behavioral defects also linked to ASD $(* p<0.05, * * * p<0.001)$.

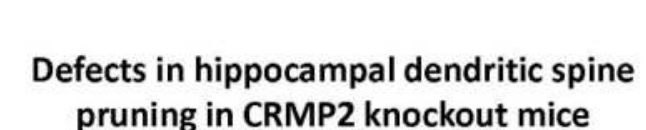
pruning in CRMP2 knockout mice
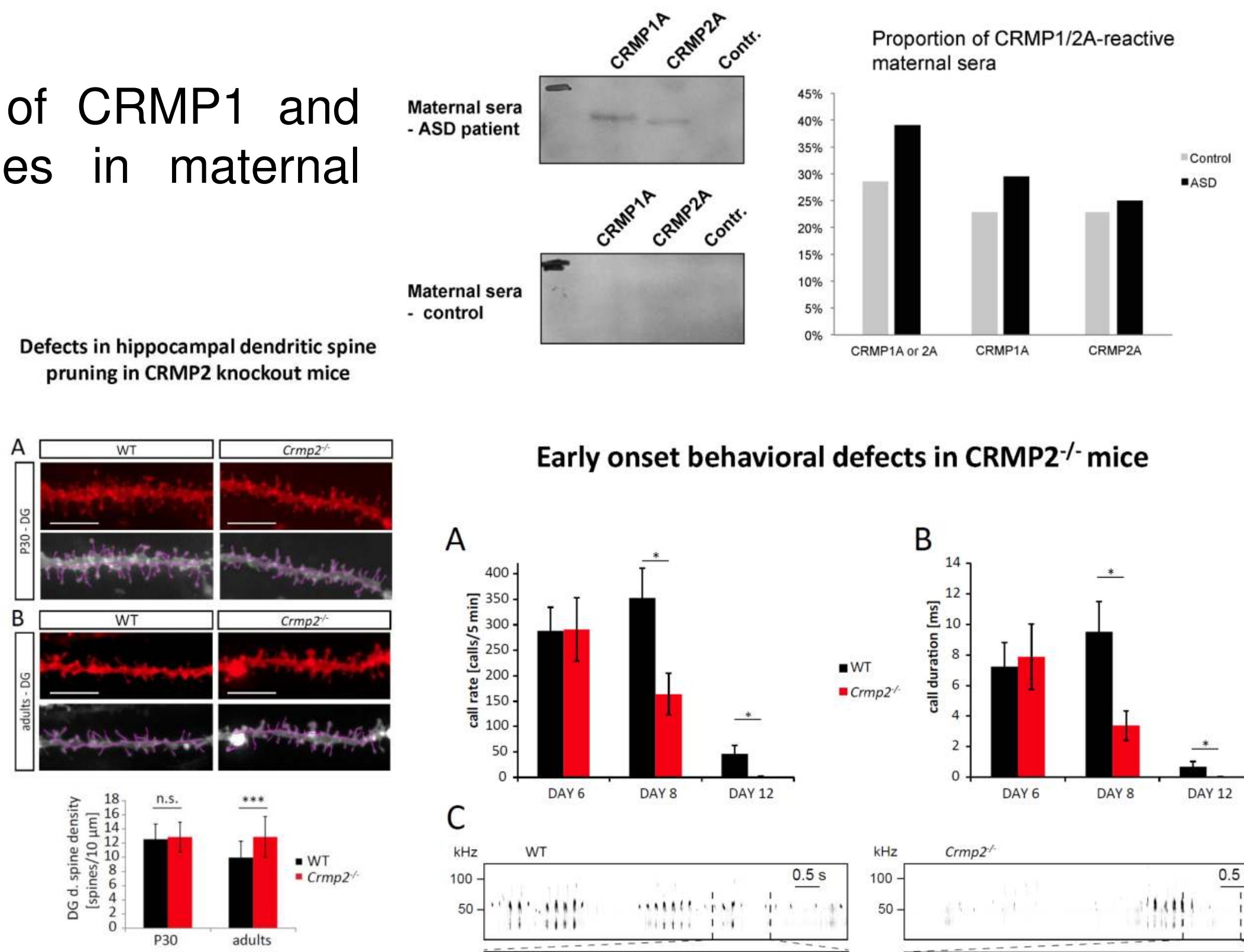

Early onset behavioral defects in CRMP2/- mice

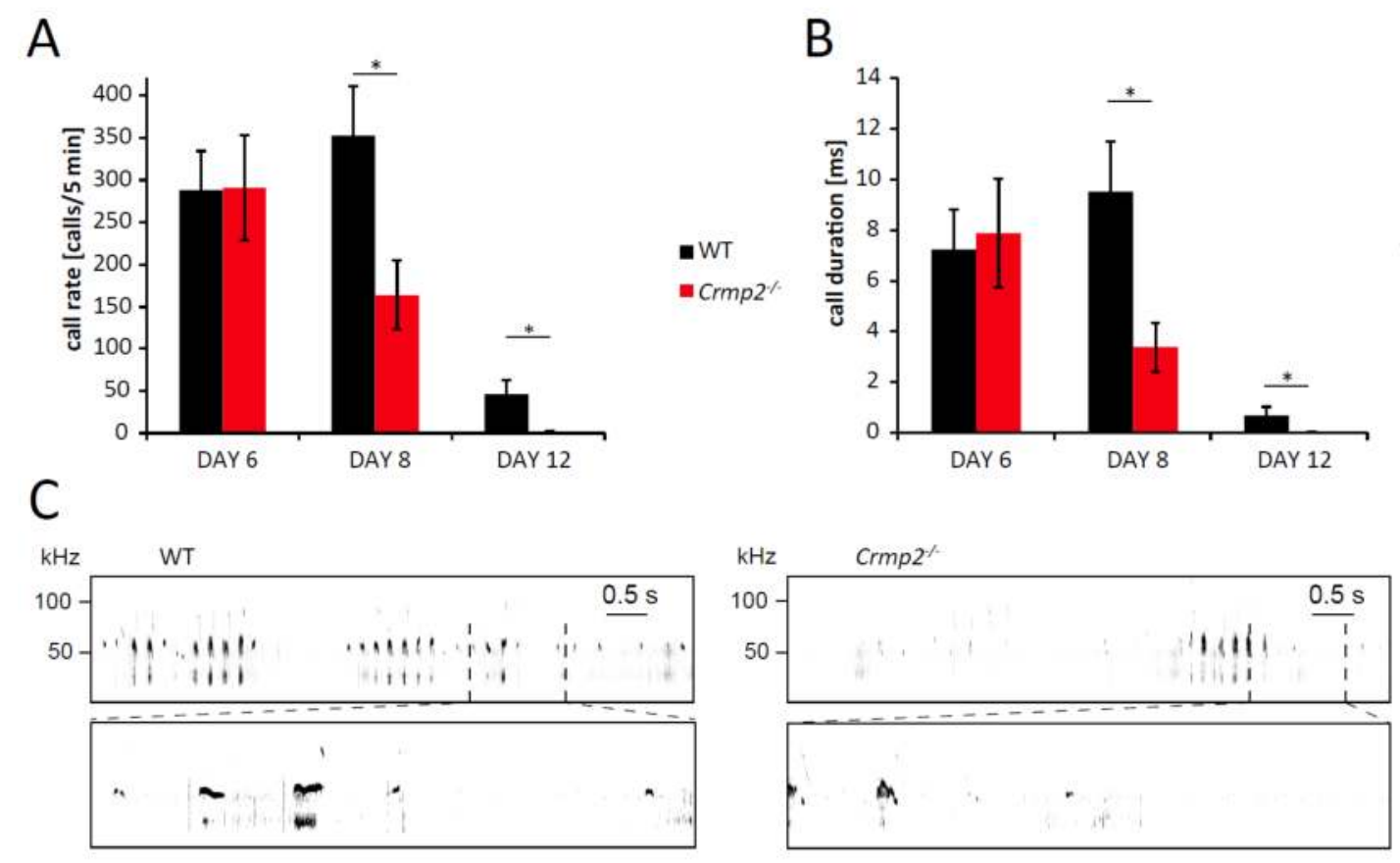

\section{Conclusion}

Our data indicate that deficiency of CRMP2 leads to ASD like phenotype in mice. As there is increased Ultrasonic vocalization

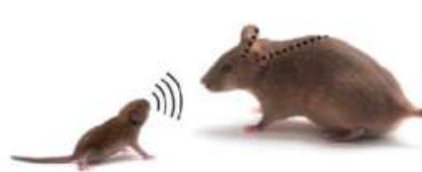

prevalence of CRMP2 and CRMP1 maternal autoantibodies in mothers of ASD patients, CRMP appears like a promising candidate gene family in ASD research. Further research will provide detail characterization of the role and the mechanism of action of CRMPs in ASD pathogenesis. 